DE

M E D I C I N A

T R O P I C A L

$\mathrm{DE}$

SÃO PAULO

JOURNAL OF THE SÃO PAULO INSTITUTE OF TROPICAL MEDICINE

${ }^{1}$ Universidade Federal de Minas Gerais, Faculdade de Farmácia, Departamento de Análises Clínicas e Toxicológicas, Belo Horizonte, Minas Gerais, Brazil

${ }^{2}$ Universidade Federal de Minas Gerais, Instituto de Ciências Biológicas, Departamento de Morfologia, Belo Horizonte, Minas Gerais, Brazil

${ }^{3}$ Instituto Nacional de Ciência e Tecnologia de Doenças Tropicais, Salvador, Bahia, Brazil

${ }^{4}$ A.C. Camargo Cancer Center, Centro Internacional de Pesquisa, São Paulo, São Paulo, Brazil

${ }^{5}$ Universidade Federal de Minas Gerais, Instituto de Ciências Exatas, Departamento de Estatística, Belo Horizonte, Minas Gerais, Brazil

6Universidade Federal de Minas Gerais Faculdade de Medicina, Programa de Pós-Graduação em Doenças Infecciosas e Medicina Tropical, Belo Horizonte, Minas Gerais, Brazil.

Correspondence to: Cristiane Alves da Silva Menezes

Universidade Federal de Minas Gerais, Faculdade de Farmácia, Departamento de Análises Clínicas e Toxicológicas, Avenida Presidente Antônio Carlos, 6627, CEP 31270-901, Belo Horizonte, MG, Brazil Tel: +55 31 3409-6874

Fax: + $55313409-6985$

E-mail: menezescristiane1@gmail.com

Received: 11 May 2018

Accepted: 14 August 2018

\section{Vasoactive intestinal peptide degradation might influence Interleukin-17 expression in cardiac chagasic patients}

\author{
Francielle Beltrão Pereira ${ }^{1}$, Walderez O. Dutra ${ }^{2,3}$, Kenneth J. Gollob ${ }^{3,4}$, Edna \\ Afonso Reis ${ }^{5}$, Ana Laura Grossi de Oliveira ${ }^{6}$, Manoel Otávio da Costa \\ Rocha $^{6}$, Cristiane Alves da Silva Menezes ${ }^{1}$
}

\section{ABSTRACT}

The vasoactive intestinal peptide (VIP) expression is lower in cardiac chagasic patients and is related to worse cardiac function. The reduction of VIP in patients with Chagas disease may be a result of its enhanced degradation. To test this hypothesis, the tryptase and chymase expression was evaluated. We also related VIP levels with interleukin-17 (IL17) expression since VIP may modulate IL-17 production. Plasma levels of chymase were higher in chagasic patients. Conversely, VIP/chymase and VIP/tryptase ratios were lower in chagasic patients when compared to non-infected individuals. Besides, the VIP/chymase ratio was lower in chagasic cardiac patients in comparison with the indeterminate group. A positive correlation between tryptase and chymase levels was observed in chagasic cardiac patients. In relation to IL-17, we observed a higher expression of this cytokine in the cardiac form of the disease than in the indeterminate form. IL-17/VIP ratio was higher in the cardiac form in comparison with non-infected or indeterminate form. These results suggest that the low levels of VIP observed in chagasic patients could be due to an increased production of chymase and/or to the additive effect of the interaction between chymase and tryptase in the cardiac form. Moreover, the decreased VIP expression may contribute to the increase of IL-17 in chagasic cardiac patients.

KEYWORDS: Chagas disease. Tryptase. Chymase. Vasoactive intestinal peptide (VIP). Interleukin-17. IL-17.

\section{INTRODUCTION}

Chagas disease is a protozoonosis caused by Trypanosoma cruzi. In endemic areas, the disease is transmitted mostly by contact with contaminated feces of Triatominae bugs. Disease can also be transmitted by blood transfusion, organ transplantation, ingestion of contaminated food and via the congenital pathway ${ }^{1,2}$.

Nowadays, it is estimated that about 7 million people worldwide, mostly in Latin America, are infected with $T$. cruzi $i^{3}$. Updated data from the American Community Survey and The World Health Organization estimated 238,000 cases across the United States $^{4}$ and in some countries in Europe as a consequence of the migratory flows of asymptomatic infected individuals from Latin America ${ }^{5}$. Remarkably, in Latin America, this parasitic disease is one of the most important of the neglected ones affecting around $13 \%$ of the population, remaining a major public health problem causing incapacity in infected individuals and more than 10,000 deaths per year ${ }^{3}$.

Once infected, the individual will develop two distinct phases: acute and chronic. During the first, trypomastigote forms of T. cruzi are abundant in the bloodstream. 
Approximately 2 months after infection, untreated/uncured individuals will develop the chronic phase of the disease, characterized by low parasitemia/low concentrations of the parasite in peripheral blood ${ }^{3}$.

The chronic phase presents distinct clinical forms. There is an asymptomatic form, called indeterminate and symptomatic forms: cardiac, digestive and cardiodigestive. The symptomatic forms of Chagas disease progress from the indeterminate status to the clinical cardiac disease and/or gastrointestinal disease. The transition from the acute phase until reaching the symptomatic forms occurs within 10 to 20 years, progressively ${ }^{6}$. There are many speculations about the factors involved in Chagas disease progression and a great interest in finding biomarkers to evidence the transition between asymptomatic to symptomatic forms.

The most significant clinical sequel of $T$. cruzi infection is chagasic cardiomyopathy. Around $20 \%$ to $30 \%$ of infected individuals will develop the cardiac clinical form, at variable time intervals after infection ${ }^{6}$. Reasons leading to the establishment of different clinical forms depend on complex interactions between the parasite and the hosts' immune system ${ }^{7}$. An uncontrolled inflammatory response can lead to the cardiac form of Chagas disease and a controlled one favors the maintenance of the indeterminate form of the disease.

Modulatory factors such as anti-inflammatory cytokines, glucocorticoids and neuropeptides are essential in controlling the inflammatory response. VIP is a neuropeptide produced by nervous and immune cells, which exerts a modulatory role over the immune response and may also act as a potent anti-trypanosome agent. Its trypanolytic activity was tested on Trypanosoma brucei and the disruption of the parasite was reached through a mechanism that involves peptides uptake by the parasite, disruption of lysosome integrity and cytosolic accumulation of glycolytic enzymes, which promotes an energetic metabolism failure that initiates an autophagiclike cell death ${ }^{8}$. Modified VIP analogues, more stable than the native peptide, were able to kill various non-pathogenic and pathogenic Gram-positive and Gram-negative bacteria, as well as the parasite Leishmania major through a mechanism that depends on the interaction with certain components of the microbial surface, the formation of pores and the disruption of the surface membrane ${ }^{9}$.

On the other hand, VIP exerts its anti-inflammatory function through its receptors, VPAC1, VPAC2 and PAC1, which are expressed by several immune cells ${ }^{10}$. VIP acts inhibiting the production of inflammatory mediators and stimulating the production of anti-inflammatory cytokines by macrophages and microglia. Additionally, VIP decreases costimulatory molecule expression in macrophages and mature dendritic cells, which will affect $\mathrm{T}$ cells stimulation. VIP also modulates $\mathrm{T}$ lymphocyte response by inhibiting the Th1 cell differentiation, favoring the expansion of Th2 cells and inducing the emergence of Treg cells (iTreg) ${ }^{11}$.

Previous studies showed that VIP is able to exert an immunomodulatory function on Th17 subpopulation, which has been appointed as pathogenic cells in several autoimmune diseases ${ }^{12,13}$. In the context of Chagas disease, Magalhães et al. ${ }^{14}$, correlating IL-17 expressing cells and cardiac function in chagasic patients, demonstrated that chagasic cardiac patients presented a lower intensity of expression of IL-17 by total lymphocytes and that a higher cellular expression of IL-17 was associated with better cardiac function. Similarly, another study suggests a relationship between high plasma expression of IL-17A and a better cardiac function in chronic human Chagas disease ${ }^{15}$. However, studies relating IL-17 and the best prognosis in Chagas disease fail in explaining the mechanism by which this cytokine would improve cardiac function.

It has been suggested that a reduction in VIP levels can contribute to the maintenance of inflammation in chronic diseases, which may lead to tissue damage ${ }^{16-19}$. We have previously shown that uninfected individuals and indeterminate Chagas patients presented a higher expression of VIP in their plasma when compared to cardiac Chagas patients, and that lower levels of VIP are associated with a worse ventricular function while higher levels of VIP are associated with better cardiac function. These data suggest a protective role to VIP in Chagas disease ${ }^{20}$.

It is possible to hypothesize that the decreased expression of VIP in cardiac patients could be related to a higher degradation rate of this neuropeptide. A classical work of Caughey et al. ${ }^{21}$, showed that mast cell proteases, tryptase and chymase, purified from dog mastocytomas are able to cleave VIP rapidly. Besides, chymase is also able to cleave another neuropeptide, named substance $P$.

The aim of this work is to measure the expression of chymase and tryptase in indeterminate and chagasic cardiac patients, and evaluate the relationship between VIP and IL-17 expression in those patients to verify if there is a correlation between the expression of these enzymes and IL-17/VIP levels and also with chagasic cardiomyopathy morbidity.

\section{MATERIALS AND METHODS}

\section{Patients}

This study was based on a cross-sectional design, employing samples from patients in endemic areas of 
Minas Gerais State, Brazil. This is an observational study, using convenience samples from chagasic patients and healthy individuals. Patients are treated in the Reference and Training Center in Infectious-Parasitic diseases of the Universidade Federal de Minas Gerais (UFMG), under the clinical care of one of the team members (MOCR). All the individuals were followed up according to standardized institutional protocols to avoid inconsistencies in classification and treatment.

Clinical classification was performed according to criteria described by Rocha et al. ${ }^{22}$. Clinical evaluation included physical examinations and exams such as: electrocardiogram, chest radiography and echocardiogram. Based on the results of these exams, patients were classified into two different clinical groups: indeterminate patients (I), asymptomatic individuals with no significant alterations in electrocardiography, chest radiography and echocardiogram; and cardiac patients (C), characterized by echocardiographic manifestations of a dilated left ventricle with impaired ventricular systolic function. We have also included a group of subjects without Chagas disease, called non-infected individuals (NI) who had negative results of the specific serological test for Chagas disease. The total of 51 biological samples included different forms of the disease: $\mathrm{I}=18 ; \mathrm{C}=14$ and $\mathrm{NI}=19$. The exclusion criteria were: presence of diabetes mellitus, arterial hypertension, renal insufficiency, thyroid dysfunction, chronic obstructive pulmonary disease, rheumatic heart diseases and any other infectious disease.

\section{Ethics}

All the participants of this study provided written informed consent. The study protocol was approved by the Research Ethics Committee of UFMG (CAAE: 18606013.7.0000.5149/COEP/UFMG-ETIC006/05) and observed the principles established in the 1975 Declaration of Helsinki revised in $1983^{23}$.

\section{Quantification of VIP, chymase (CMA) and tryptase (TPS) by ELISA}

Blood samples were collected from the three different groups: chagasic patients $(I=18, C=14)$ and non-infected individuals $(\mathrm{NI}=19)$. Samples were collected in EDTA tubes and centrifuged to recover plasma samples. VIP, CMA and TPS plasma levels were measured using specific ELISA kits (Uscn Life Science, Huston, TX). The manufacturer's protocols were followed. Plates were read in a microplate spectrophotometer at $450 \mathrm{~nm}$ and results were analyzed based on standard curves.

\section{Quantification of IL-17A}

A cytometric bead array (CBA), a bead-based immunoassay capable of measuring human cytokines in serum, plasma and cell culture supernatant samples was used (Becton Dickinson Biosciences, San Jose, CA, USA) to quantify plasma IL-17A expression. The test was performed according to the manufacturer's instructions. Data were acquired in a BD LSRFortessa flow cytometry system and analyses were performed using the BD FCAP Array v3.0 software. Results were based on a standard concentration curve and expressed as picograms per milliliter $(\mathrm{pg} / \mathrm{mL})$.

The detection limit to IL-17 was $18.9 \mathrm{pg} / \mathrm{L}$ and results below this detection limit were excluded.

\section{Statistical analysis}

Data were analyzed for normal distribution by the Shapiro-Wilk test. Nonparametric data were analyzed by the Kruskall-Wallis for comparisons among the three groups. The Mann-Whitney test was used for comparison of two groups. Correlation analyses were performed using the Spearman linear correlation coefficient and squares regression. Results were nominally significant at two-sided $p<0.05$. Statistical analysis of data was performed by using the GraphPad Prism statistical software (version 6; San Diego, CA).

\section{RESULTS}

\section{VIP, chymase and tryptase plasma levels}

VIP, chymase and tryptase plasma levels were evaluated in the groups I $(\mathrm{n}=18), \mathrm{C}(\mathrm{n}=14)$ and NI $(\mathrm{n}=19)$. VIP plasma levels were significantly lower in cardiac patients $(5.1 \pm 0.7 \mathrm{pg} / \mathrm{mL})$, when compared to non-infected individuals $(11.1 \pm 1 \mathrm{pg} / \mathrm{mL})$ and indeterminate patients $(8.2 \pm 0.9 \mathrm{pg} / \mathrm{mL})$ (Figure 1A). In relation to chymase plasma levels, it was observed that chagasic patients from groups I $(15.6 \mathrm{ng} / \mathrm{mL})$ and C $(24.81 \mathrm{ng} / \mathrm{mL})$ forms presented a median of chymase 3-4 times higher when compared to the NI group (6.8 ng/ $\mathrm{mL} ; \mathrm{p}<0.001$ for both comparisons) (Figure $1 \mathrm{~A}$ ). There was no difference in the median of plasma chymase expression between chagasic patients of the two different clinical forms (Figure 1A). The median of tryptase plasma levels was not statistically different between the groups NI $(31.2 \mathrm{ng} / \mathrm{mL})$, I $(42.32 \mathrm{ng} / \mathrm{mL})$ and $\mathrm{C}(32.59 \mathrm{ng} / \mathrm{mL})$ individuals (Figure 1B).

Ratio of vasoactive intestinal peptide and chymase or tryptase

We have previously shown that Chagas patients 


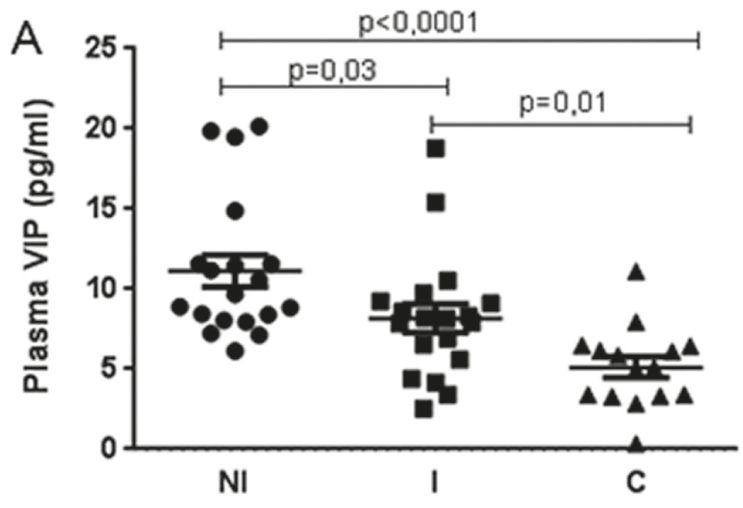

B
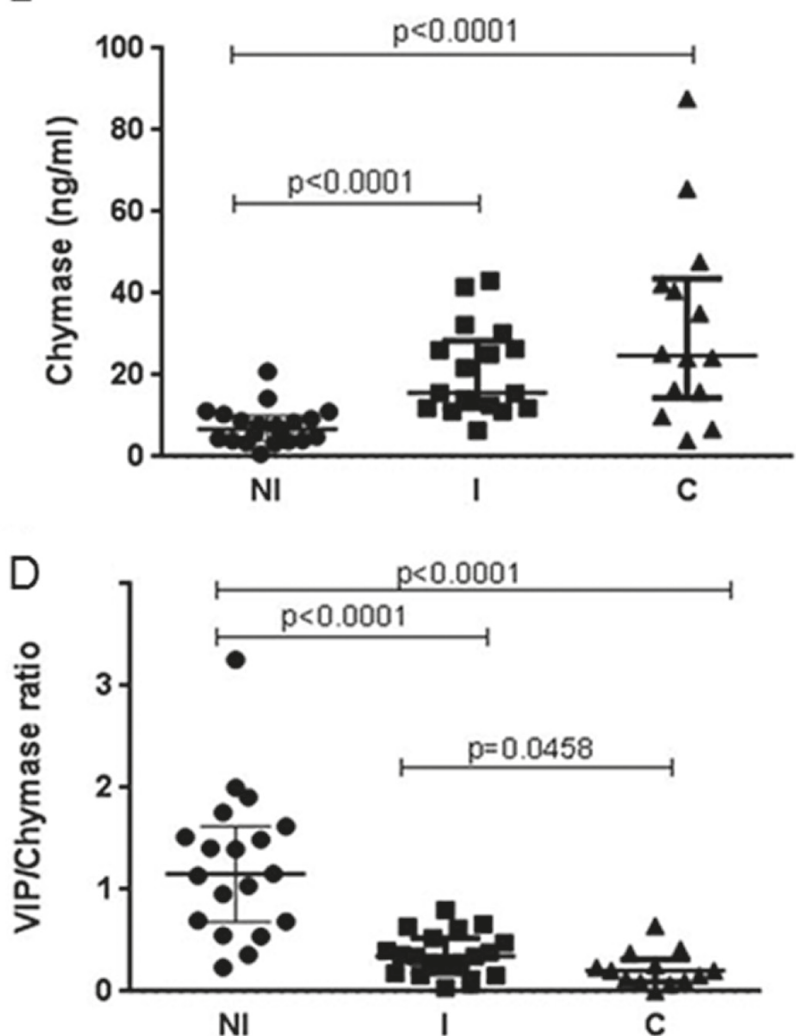

C

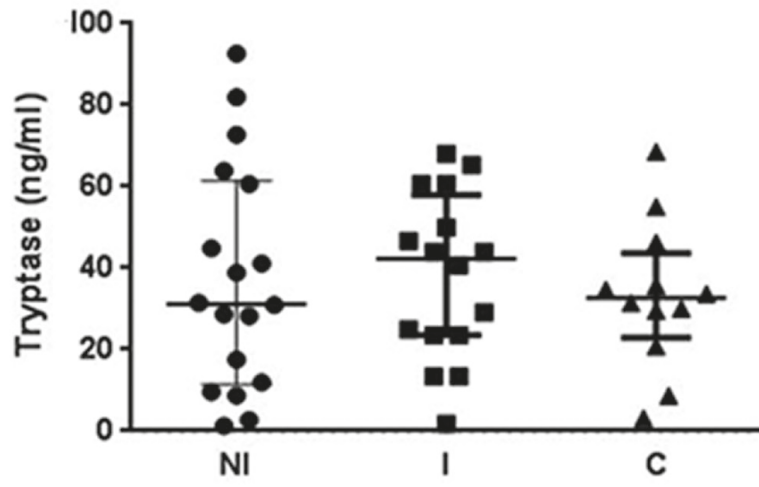

E

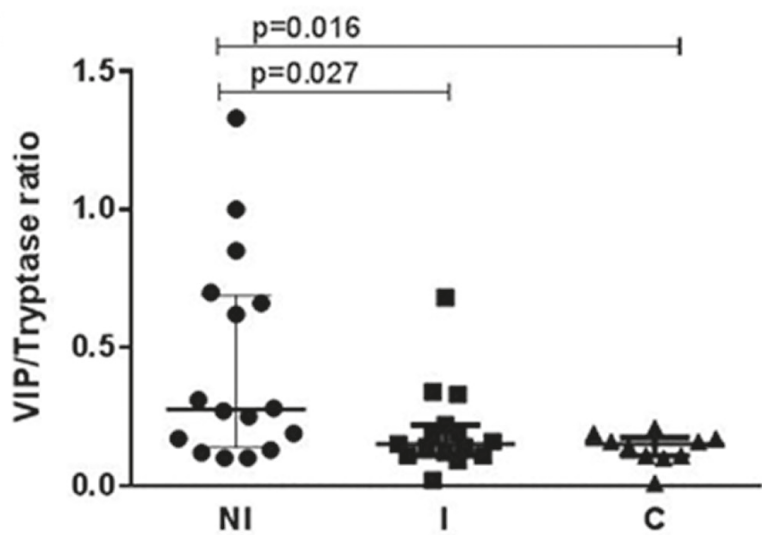

Figure 1 - Plasma levels of Vasoactive Intestinal Peptide (VIP) Chymase (CMA), Tryptase (TPS) and VIP/Chymase (CMA) or VIP/Tryptase (TPS) ratios in chagasic patients. A) Plasma levels of VIP in non-infected individuals (NI), indeterminate patients (I) and cardiac patients (C). Plasma levels of TPS in non-infected individuals (NI) indeterminate patients (I) and cardiac individuals (C); B) Plasma levels of CMA in non-infected individuals ( $\mathrm{NI}$ ), indeterminate patients (I) and cardiac patients (C); C) Plasma levels of TPS in non-infected individuals (NI) indeterminate patients (I) and cardiac individuals (C); D) VIP/CMA ratio in non-infected individuals $(\mathrm{NI})$ indeterminate patients $(\mathrm{I})$ and cardiac individuals $(\mathrm{C})$; E) VIP/TPS ratio in non-infected individuals (NI) indeterminate patients (I) and cardiac individuals (C). Q1, Q3 and median values are shown. Significant differences between groups are indicated by connecting lines. Kruskal-Wallis test followed by Mann-Withney test $(p<0.05)$ were used for comparisons.

(indeterminate and cardiac) had lower levels of VIP compared to controls and that cardiac patients had lower levels of this peptide in relation to indeterminate patients ${ }^{20}$ Thus, we evaluated the ratio of VIP, chymase and tryptase considering that the unbalance between VIP production and VIP degradation might be a determining factor contributing to the lower levels of VIP in those patients.
It was observed that VIP/CMA median ratio was lower in indeterminate $(0.35 ; \mathrm{p}<0.0001)$ and cardiac $(0.21 \mathrm{ng} / \mathrm{mL}$; $\mathrm{p}<0.0001)$ patients when compared to non-infected $(1.16 \mathrm{ng} / \mathrm{mL}$ ) individuals (Figure 1C). VIP/CMA ratio was also different between indeterminate and cardiac groups ( $\mathrm{p}=0.0458$ ) (Figure 1C). In relation to the VIP/ TPS median ratio, a statistically significant difference 

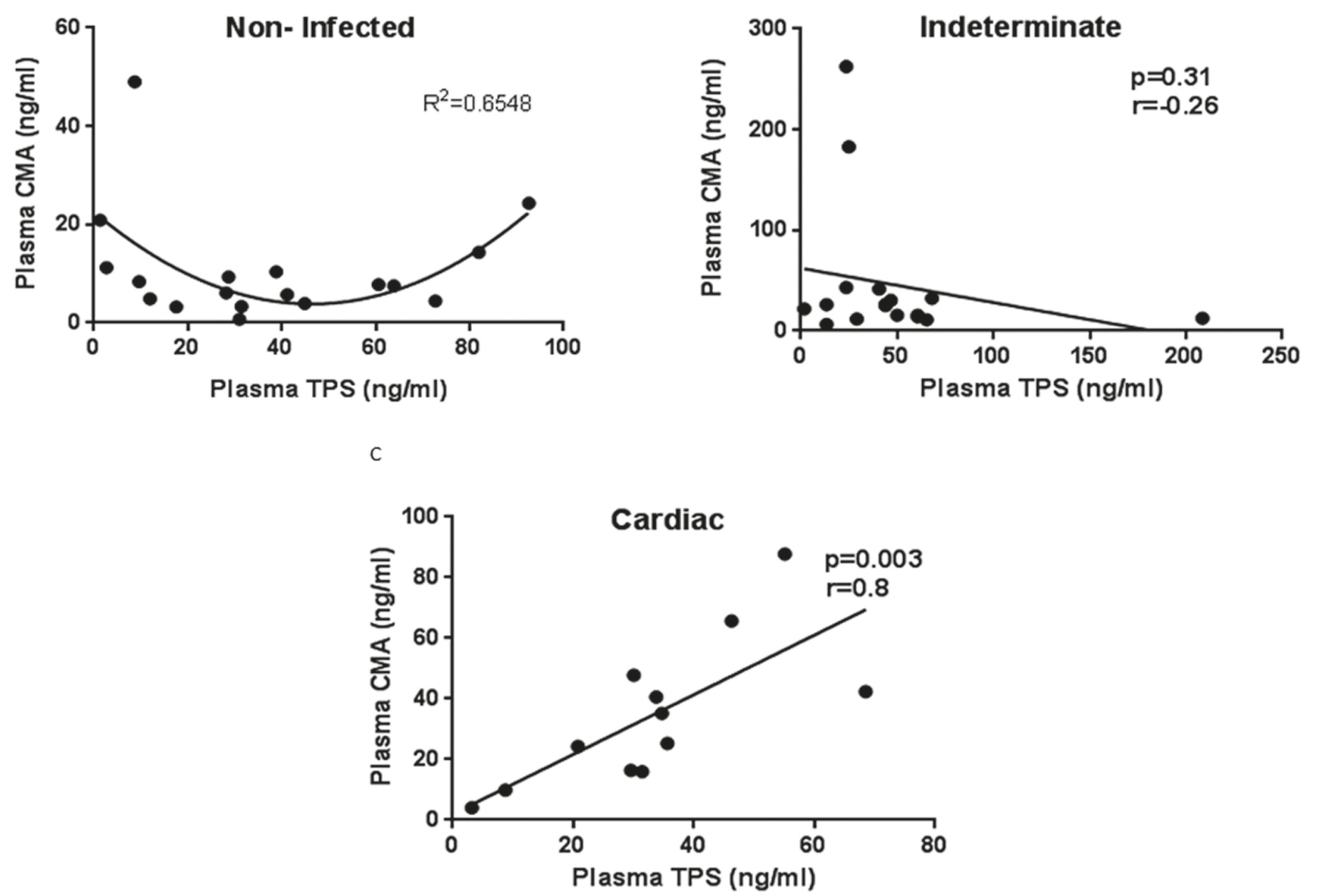

Figure 2 - Correlation analysis between plasma levels of CMA and TPS in chagasic patients. A) Correlation analysis between CMA and TPS plasma levels in non-infected individuals; B) Correlation analysis between CMA and TPS plasma levels in the indeterminate group; C) Correlation analysis between CMA and TPS plasma levels in the cardiac group. Statistical analyses were performed by using the square regression coefficient of determination $\left(R^{2}\right)$ for the NI group and the Spearman correlation coefficient $(r)$ for I and $\mathrm{C}$ groups.

was observed between indeterminate $(0.14 ; \mathrm{p}=0.027)$ and cardiac patients $(0.15 ; \mathrm{p}=0.012)$ and non-infected $(0.28 \mathrm{ng} / \mathrm{mL})$ individuals (Figure 1D). VIP/TPS median ratio was not different in the indeterminate $(0.14 \mathrm{ng} / \mathrm{mL})$ individuals when compared to the cardiac $(0.15 \mathrm{ng} / \mathrm{mL})$ patients (Figure 1D).

\section{Correlation analysis between chymase and tryptase}

Since the peptidases are interdependent, the absence or inactivity of one enzyme can alter levels and activity of others ${ }^{24}$. As CMA and TPS are able to degrade VIP, we performed a correlation analysis between the levels of both enzymes in each studied group in order to observe if their expressions are related. A square relation between chymase and tryptase was observed in non-infected individuals. Therefore, values of TPS close to $40 \mathrm{ng} / \mathrm{mL}$ are followed by a reduction of CMA and values of TPS higher than $40 \mathrm{ng} / \mathrm{mL}$ are followed by an increase of CMA values $\left(R^{2}=0.65\right)$ (Figure 2A). In indeterminate individuals, a correlation was not observed between CMA and TPS expression $(\mathrm{r}=-0.26$; $\mathrm{p}=0.31)$ (Figure 2B). Interestingly, a positive linear correlation was observed between CMA and TPS levels in the cardiac group $(r=0.8 ; p=0.003)$, indicating that higher levels of chymase were related with higher tryptase levels in those individuals (Figure 2C). Correlation analyses between CMA and TPS versus VIP were performed, however, they did not present any statistical significances (data not shown).

\section{Ratio of vasoactive intestinal peptide and IL-17}

IL-17 plasma levels were evaluated in indeterminate chagasic patients $(n=10)$, chagasic cardiac patients $(n=10)$ and non-infected individuals $(\mathrm{n}=14)$. In relation to IL-17 plasma levels, it was observed that cardiac Chagas patients presented higher levels of this cytokine $(140.1 \mathrm{pg} / \mathrm{mL})$ than the indeterminate patients $(22.9 \mathrm{pg} / \mathrm{mL}$; $\mathrm{p}=0.0047)$ and non-infected individuals $(57.6 \mathrm{pg} / \mathrm{mL}$; $\mathrm{p}=0.056)$. The production of IL-17 was also lower in indeterminate patients when compared to healthy individuals $(\mathrm{p}=0.043)$ (Figure $3 \mathrm{~A})$. In relation to 
A

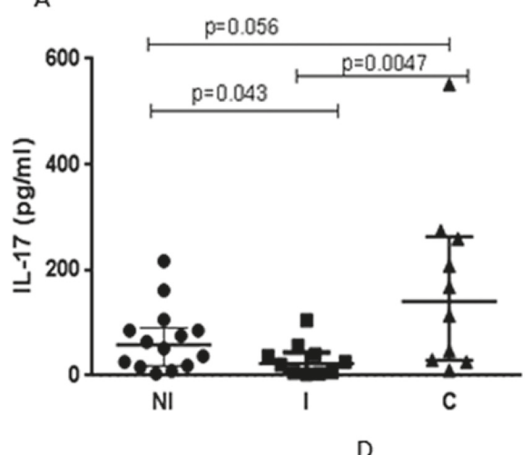

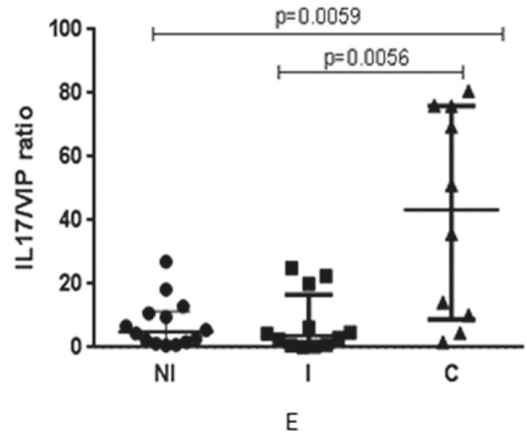
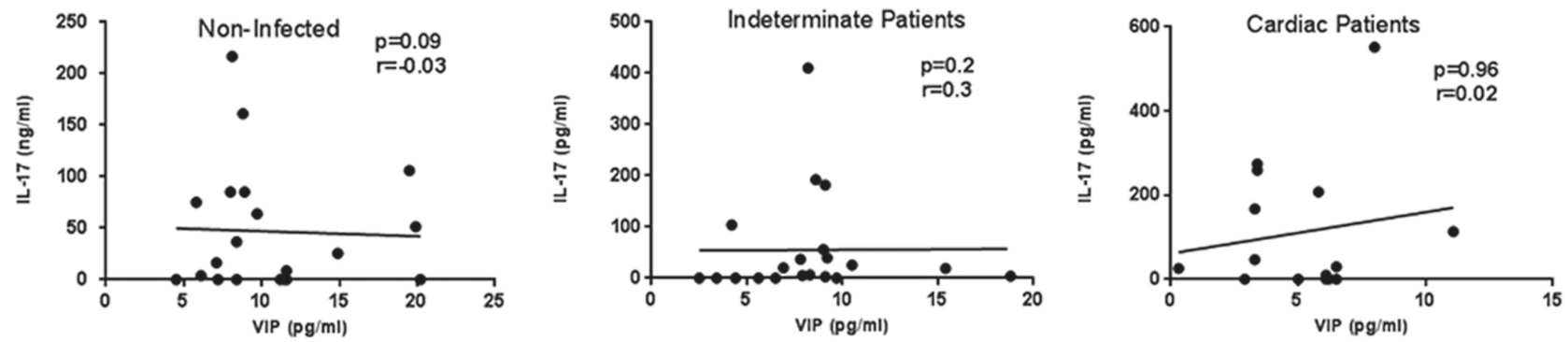

Figure 3 - Analyses of plasma IL-17, IL-17/VIP ratio and IL-17/VIP correlation in chagasic patients. A) IL-17 plasma levels of noninfected (NI), indeterminate patients (I) and cardiac individuals (C); B) Plasma IL-17/VIP ratio of non-infected (NI), indeterminate patients (I) and cardiac individuals (C); C) Correlation analysis between IL-17 and VIP plasma levels in non-infected individuals; D) Correlation analysis between IL-17 and VIP plasma levels in the indeterminate group; E) Correlation analysis between IL-17 and VIP plasma levels in the cardiac group. Q1, Q3 and median values are shown. Significant differences between groups are indicated by connecting lines. Kruskal-Wallis test followed by Mann-Withney test $(p<0.05)$ were used for comparisons. Correlation analyses were performed by using the Spearman correlation coefficient $(r)$.

IL-17/VIP ratio, a higher ratio was observed in chagasic cardiac patients (43.31) when compared to indeterminate (3.6; $\mathrm{p}=0.0056)$ and non-infected individuals (4.9; $\mathrm{p}=0.0059)$ (Figure 3B). Correlation analyses between IL-17 versus VIP were performed; however, they did not present statistical significances (Figure 3C-3E).

\section{DISCUSSION}

VIP is a neuropeptide constituted by 28 amino acids and presents a wide body distribution. VIP exerts a role as a modulator of innate and adaptive immunity, being a potent anti-inflammatory agent. VIP receptors, VPAC1 and VPAC2 are expressed by several immunocompetent cells. The engagement of VIP with its G protein-coupled receptors activates $\mathrm{AMPc} / \mathrm{PKA}$ and, consequently, activates transcription factors ${ }^{25}$ especially those favoring the antiinflammatory pathways. A higher expression of VIP has been related to a better prognosis in different chronic diseases ${ }^{16-19}$.

This work and previous studies showed that VIP plasma levels are higher in non-infected individuals when compared to chagasic patients of indeterminate and cardiac clinical forms ${ }^{20}$. One hypothesis to explain the decrease of VIP expression in chagasic patients could be an increased expression of enzymes with VIPase activity. Mast cells produce and secrete many peptidases, such as chymase and tryptase, which are able to degrade VIP. Tryptase cleaves VIP rapidly at two sites and chymase cleaves VIP primarily at a single site ${ }^{21}$. Differences in peptidase activity between tryptase and chymase suggest that the consequences of protease release could vary according to the mast cell protease phenotype and the location in various tissues and species $^{21}$.

Results of the present work indicate that the lower expression of VIP in chagasic patients might be related to a higher expression of chymase. In addition, VIP/chymase ratio was lower in chagasic patients when compared to uninfected individuals and, the same ratio was also lower in cardiac patients when compared to the indeterminate form. Both results corroborate the hypothesis that chymase might contribute to the observed decrease of VIP levels detected in chagasic patients.

Tryptase expression was not significantly different amongst the groups analyzed. Correlation analyses between chymase and tryptase were performed. An equilibrium between the expressions of both enzymes was observed for non-infected individuals. In indeterminate patients, chymase/tryptase equilibrium expression seems to be lost. In chagasic cardiac patients, the higher the expression 
of chymase, the greater the expression of tryptase. It is known that peptidases might influence the expression levels and activity of others ${ }^{24}$, thus it is likely that the combined action of both enzymes may result in a decrease of VIP levels observed in cardiac patients. Moreover, these enzymes have a role in the inflammatory process, influencing leukocytes chemotaxis, lymphocytes activation, expression and release of proinflammatory chemokynes and cytokynes, leading to immunoregulatory disorders ${ }^{26-28}$. Altogether, chymase and tryptase may be related to cardiac damage in chagasic cardiac disease not only by decreasing VIP levels, but also by contributing to the exacerbation of inflammatory responses in cardiac patients. On the other hand, indeterminate individuals, despite presenting higher chymase levels and reduction of VIP/chymase ratio when compared to non-infected individuals, did not show a positive correlation between chymase and tryptase expression, which may indicate that in asymptomatic chagasic individuals, the enzymes do not present additive effects. This may be an important mechanism to maintain immune modulation, avoiding the occurrence of tissue damage in these patients. There are few studies evaluating the expression of VIP and its degrading proteases in cardiac Chagas disease. In the digestive form of Chagas disease, a higher expression of tryptase was found in patients presenting megaesophagus when compared to individuals without it or uninfected ones, suggesting that tryptase could participate in the megaesophagus development in infected individuals. The pro-inflammatory effects of this enzyme and its role in VIP degradation may be part of the mechanisms associated with the development of these severe digestive alterations ${ }^{29,30}$. Differences between those studies may rely on several characteristics such as: clinical forms; clinical characterization of patients; type of samples used; genetic and immune characteristics of patients and genetic variability of the parasite. Despite all these variables, all studies are valid and non-exclusive and show a clear correlation between lower VIP expression and disease severity.

An in situ study evaluated the expression of chymase and tryptase by mast cells of individuals presenting digestive forms of Chagas disease. A higher concentration of mast cells reactive to tryptase was observed in Chagas individuals presenting megaesophagus or not when compared to noninfected individuals. In relation to chymase expression by mast cells, a higher expression was observed only in infected individuals without megaesophagus ${ }^{30}$. Our results showed a higher expression of chymase in cardiac and indeterminate Chagas disease patients when compared to non-infected individuals. Moreover, we observed no differences of tryptase expression among the analyzed groups. It is possible that in digestive forms, tryptase is responsible for VIP degradation. Interestingly, chymase is involved in the development of cardiovascular diseases in animal models ${ }^{31}$.

In humans, a positive correlation between the collagen expression and chymase expressing mast cells was observed in the myocardium of chagasic patients suggesting that chymase may also be associated with fibrosis in cardiac Chagas disease ${ }^{32}$. It is possible that chymase expression in cardiac forms of Chagas disease, might be related to the aggravation of the cardiac disease, possibly by its VIPase, proinflammatory, vascular and fibrosing effects. Additionally, the tryptase expression seems to be coordinated with the chymase one, in cardiac patients, who might potentiate the chymase function.

Lower VIP levels have been related to worse prognosis, not only in Chagas disease ${ }^{20}$ but also in other chronic diseases. A study evaluated serum levels of VIP during the follow-up of an early arthritis (EA) cohort ${ }^{33}$. Patients fulfilling the criteria for rheumatoid arthritis (RA) presented the lowest values of VIP although no significant differences were observed in comparison with healthy donors. In addition, the disease activity was inversely correlated with VIP levels. After a two-year follow-up, patients with low baseline levels of VIP displayed higher disease activity. Another study analyzed the correlation between serum levels of VIP and disease activity/severity in patients with early chronic inflammatory back pain associated with Spondyloarthritis (SpA). Lower levels of VIP were significantly associated with a higher Bath Ankylosing Spondylitis Disease Activity Index (BASFI) score, presence of bone edema in magnetic resonance imaging (MRI) scans and lower hemoglobin levels. Results indicated that patients with low serum VIP levels had worse 2-year disease outcome ${ }^{34}$. Another study evaluating VIP levels in patients with osteoarthritic knee (OA), observed that OA patients had lower VIP concentrations in synovial fluid and articular cartilage. Moreover, the synovial fluid and articular cartilage lower levels of VIP were negatively correlated with disease severity ${ }^{35}$. All these studies indicate that lower VIP levels are related to a poor prognosis of the diseases and highlight the possibility of using VIP levels measurements as a prognostic biomarker.

The present work also evaluated if VIP expression may have some impact on IL-17 plasma expression since a decrease of Th1 and Th17 profiles is one of the VIP effects ${ }^{36}$. Th17 cells may present a pathogenic or non-pathogenic phenotype according to their cytokine secretion profile and VIP reduces the pathogenic profile of Th17-polarized cells $^{13,37,38}$. 
Some studies have been developed focusing on Th17 population and/or IL-17 expression in cardiovascular diseases. An increase of peripheral Th17 cells and Th17 related cytokines was observed in patients presenting dilated cardiomyopathy ${ }^{38,39}$. In Chagas disease, a recent study has analyzed the frequency of $\mathrm{CD} 4{ }^{+} \mathrm{CD} 25^{+} \mathrm{FoxP} 3^{+}$cells, classic Th17 cells, alternative Th17 cells and $\mathrm{IL}-17^{+} \mathrm{B}$ cells from peripheral blood of chronic cardiac patients after in vitro stimulation with $T$. cruzi antigen. The results showed that $\mathrm{CD}^{+} \mathrm{CD} 25^{+} \mathrm{FoxP}^{+}, \mathrm{CD} 4^{+} \mathrm{CD} 25^{\text {high }} \mathrm{FoxP}^{+}, \mathrm{CD} 4^{+} \mathrm{IL}-17^{+}$ IFN- $\gamma$ and $\mathrm{CD} 4^{+}$IL- $17^{+}$IFN- $\gamma^{+}$cells are more frequent in patients with severe cardiac disease and these findings correlate with the worsening of global cardiac function. IL-17 expression by Th17 cells and B cells correlated with disease progression. These results suggest that the clinical progression of Chagas cardiomyopathy involves worsening of inflammation and impairment of immunoregulatory mechanisms with IL-17 participation ${ }^{40}$.

Regarding Chagas disease, a report on the role of IL-17 and regulatory $\mathrm{T}$ cells in human Chagas disease showed higher percentages of CD4 $4^{+} \mathrm{IL}-17^{+}$cells in PBMC cultured from patients with or without mild cardiomyopathy, in comparison with patients presenting moderate or severe cardiomyopathy ${ }^{41}$. The study also showed that $\mathrm{CD} 4^{+} \mathrm{CD} 25^{+} \mathrm{T}$ cells from patients with mild or without cardiomyopathy presented higher suppressive activity than those with moderate and severe cardiomyopathy. The authors concluded that deficient regulatory $\mathrm{T}$ cell activity and low frequency of IL-17-producing T cells correlate with the extent of cardiomyopathy in human Chagas disease ${ }^{41}$. Another study investigated the intracellular IL17 expression and its association with the indeterminate or cardiac clinical forms of Chagas disease and with patients' cardiac function ${ }^{14}$. Their results demonstrated that cardiac patients presented a lower intensity of IL-17 expression by total lymphocytes and a lower frequency of circulating $\mathrm{T}$ helper 17 cells. Another study evaluating the expression of IL-17 in plasma samples of Chagas patients ${ }^{15}$ observed that a significant frequency of indeterminate patients presented high levels of IL-17A, whereas most of the cardiac patients expressed low plasma levels of this cytokine. Thus, the authors have shown that low and high IL-17A producers were observed in both groups. A recent study investigated the associations between genetic polymorphisms of IL17A G197A and IL17F T7488C with Chagas disease (CD) and/or the severity of left ventricular systolic dysfunction (LVSD). The analysis according to the gender showed that the A/A genotype of IL17A was more frequent in female patients with LVSD and mild to moderate LVSD, and also in male patients with LVSD. The frequency of IL17F T/C genotype was higher in male patients with CCC and severe LVSD and in females with mild to moderate LVSD. The mutant allele A of IL17A was associated with a higher production of IL-17 and it is known that IL-17F activity is similar to IL-17A. Based on these findings, it is possible to infer that the higher production of IL-17 could contribute to tissue damage and might be related to the development and progression of $\mathrm{CCC}^{42}$. In our study, we observed a higher plasma expression of IL-17A in cardiac patients when compared to indeterminate ones. However, our study presented a restrict sample size in relation to the cited study and, it seems that most of our patients belonged to the high IL-17A producers group. In relation to IL-17/VIP ratio, we observed a higher ratio in chagasic cardiac patients when compared with indeterminate and non-infected individuals. It is a direct effect of the elevated expression of IL-17 in the cardiac group analyzed by us and the lower expression of VIP in those patients. This result may indicate a lack of modulation of Th17 cells due to decreased VIP expression, which may cause these cells to develop a pathogenic profile that, in this cardiac group, may contribute to heart damage. Studies to define the profile of Th17 cells in clinical forms of Chagas disease are necessary.

In summary, our results suggest that the ratio between production/degradation of VIP is compromised in chagasic patients, and the unbalanced levels of VIP may have implications on IL-17 expression. Future studies are necessary to point out how VIP and VIPase enzymes influence the immune system response in chronic inflammatory diseases, which could allow the proposition of new treatment strategies and/or new therapeutic associations aiming the improvement of patients' management.

\section{ACKNOWLEDGMENTS}

This work was supported by grants from FAPEMIG (Demanda Universal/2015) and PRPq-UFMG. WOD, KJG and MOCR are CNPq fellows.

\section{FINANCIAL SUPPORT}

\section{FAPEMIG and CNPq.}

\section{REFERENCES}

1. Schorderet-Weber S, Noack S, Selzer PM, Kaminsky R. Blocking transmission of vector-borne diseases. Int J Parasitol Drugs Drug Resist. 2017;7:90-109.

2. Dias JC, Amato Neto V, Luna EJ. Mecanismos alternativos de transmissão do Trypanosoma cruzi no Brasil e sugestões para sua prevenção. Rev Soc Bras Med Trop. 2011;44:375-9. 
3. World Health Organization. Chagas disease (American trypanosomiasis). [cited 2017 Nov 8]. Available from: http:// www.who.int/mediacentre/factsheets/fs340/en/

4. Manne-Goehler J, Umeh CA, Montgomery SP, Wirtz VJ. Estimating the burden of Chagas disease in the United States. PLoS Negl Trop Dis. 2016;10:e0005033.

5. Antinori S, Galimberti L, Bianco R, Grande R, Galli M, Corbellino M. Chagas disease in Europe: a review for the internist in the globalized world. Eur J Intern Med. 2017;43:6-15.

6. Menezes CA, Chaves AT, Rocha MO. Pathogenicity of Chagas disease cardiopathy. JSM Atheroscler. 2016;1:1009.

7. Rocha MO, Nunes MC, Ribeiro AL. Morbidity and prognostic factors in chronic chagasic cardiopathy. Mem Inst Oswaldo Cruz. 2009;104 Suppl 1:159-66.

8. Delgado M, Anderson P, Garcia-Salcedo JA, Caro M, GonzalezRey E. Neuropeptides kill African trypanosomes by targeting intracellular compartments and inducing autophagic-like cell death. Cell Death Differ. 2009;16:406-16.

9. Campos-Salinas J, Cavazzuti A, O’Valle F, Forte-Lago I, Caro M, Beverley AM, et al. Therapeutic efficacy of stable analogues of vasoactive intestinal peptide against pathogens. J Biol Chem. 2014;289:14583-99.

10. Ganea D, Delgado M. The neuropeptides VIP/PACAP and T cells: inhibitors or activators? Curr Pharm Des. 2003;9:997-1004.

11. Deng S, Xi Y, Wang H, Hao J, Niu X, Li W, et al. Regulatory effect of vasoactive intestinal peptide on the balance of Treg and Th17 in collagen-induced arthritis. Cell Immunol. 2010;265:105-10.

12. Ganea D, Hooper KM, Kong W. The neuropeptide vasoactive intestinal peptide: direct effects on immune cells and involvement in inflammatory and autoimmune diseases. Acta Physiol (Oxf). 2015;213:442-52.

13. Jimeno R, Leceta J, Martínez C, Gutiérrez-Cañas I, Pérez-García $\mathrm{S}$, Carrión M, et al. Effect of VIP on the balance between cytokines and master regulators of activated helper $\mathrm{T}$ cells. Immunol Cell Biol. 2012;90:178-86.

14. Magalhães LM, Villani FN, Nunes MC, Gollob KJ, Rocha MO, Dutra WO. High interleukin 17 expression is correlated with better cardiac function in human Chagas disease. J Infect Dis. 2013;207:661-5.

15. Sousa GR, Gomes JA, Damasio MP, Nunes MC, Costa HS, Medeiros NI, et al. The role of interleukin 17-mediated immune response in Chagas disease: high level is correlated with better left ventricular function. PLoS One. 2017;12:e0172833.

16. Sun W, Hong J, Zang YC, Liu X, Zhang JZ. Altered expression of vasoactive intestinal pepetide receptors in T lymphocytes and aberrant Th1 immunity in multiple sclerosis. Int Immunol. 2006;18:1691-700.

17. Anderson P, Delgado M. Endogenous anti-inflammatory neuropeptides and pro-resolving lipid mediators: a new therapeutic approach for immune disorders. J Cell Mol Med. 2008; $12: 1830-47$.
18. Delgado M, Robledo G, Rueda B, Varela N, O'Valle F, Hernandez-Cortes $\mathrm{P}$, et al. Genetic association of vasoactive intestinal peptide receptor with rheumatoid arthritis: altered expression and signal in immune cells. Arthritis Rheum. 2008;58:1010-9.

19. Prasse A, Zissel G, Lützen N, Schupp J, Schmiedlin R, GonzalezRey E, et al. Inhaled vasoactive intestinal peptide exerts immunoregulatory effects in sarcoidosis. Am J Respir Crit Care Med. 2010;182:540-8.

20. Corrêa MV, Rocha MO, Sousa GR, Nunes MC, Gollob KJ, Dutra WO, et al. Low levels of vasoactive intestinal peptide are associated with Chagas disease cardiomyopathy. Hum Immunol, 2013;74:1375-81.

21. Caughey GH, Leidig F, Viro NF, Nadel JA. Substance P and vasoactive intestinal peptide degradation by mast cell tryptase and chymase. J Pharmacol Exp Ther. 1988;244:133-7.

22. Rocha MO, Teixeira MM, Ribeiro AL. An update on the management of Chagas cardiomyopathy. Expert Rev Anti Infect Ther. 2007;5:727-43.

23. World Medical Association. World Medical Association Declaration of Helsinki: ethical principles for medical research involving human subjects. JAMA. 2013;310:2191-4.

24. Trivedi NN, Caughey GH. Mast cell peptidases: chameleons of innate immunity and host defense. Am J Respir Cell Mol Biol. 2010;42:257-67.

25. Gonzalez-Rey E, Delgado M. Anti-inflammatory neuropeptide receptors: new therapeutic targets for immune disorders? Trends Pharmacol Sci. 2007;28:482-91.

26. Bangale Y, Karle S, Planque S, Zhou YX, Taguchi H, Nishiyama Y, et al. VIPase autoantibodies in FAs-defective mice and patients with autoimmune disease. FASEB J. 2003;17:628-35.

27. Juarranz Y, Gutiérrez-Cañas I, Santiago B, Carrión M, Pablos JL, Gomariz RP. Differential expression of vasoactive intestinal peptideand its functional receptors in human osteoathritic and rheumatoid synovial fibroblasts. Atrthrits Rheum. 2008;58:1086-95.

28. Gulubova MV. Structural examination of tryptase- and chymasepositive mast cells in livers, containing metastases from gastrointestinal cancers. Clin Exp Metastasis. 2003;20:611-20.

29. Nascimento RD, Martins PR, Lisboa AS, Adad SJ, Silveira AB, Reis DA. An imbalance between substance P and vasoactive intestinal polypeptide might contribute to the immunopathology of megaesophagus after Trypanosoma cruzi infection. Hum Pathol. 2013;44:269-76.

30. Martins PR, Nascimento RD, Lisboa AS, Martinelli PM, Reis DA. Neuroimmunopathology of Trypanosoma cruzi-induced megaoesophagus: is there a role for mast cell proteases? Hum Immunol. 2014;75: 302-5.

31. Takai S, Jin D, Miyazaki M. Chymase as an important target for preventing complications of metabolic syndrome. Curr Med Chem. 2010;17:3223-9. 
32. Roldão JA, Beghini M, Ramalho LS, Porto CS, Rodrigues DB, Teixeira VP, et al. Comparison between the collagen intensity and mast cell density in the lingual muscles and myocardium of autopsied chronic chagasic and nonchagasic patients. Parasitol Res. 2012;111:647-54.

33. Martínez C, Ortiz AM, Juarranz Y, Lamana A, Seoane IV, Leceta $\mathrm{J}$, et al. Serum levels of vasoactive intestinal peptide as a prognostic marker in early arthritis. PloS One. 2014;9:e85248.

34. Seoane IV, Tomero E, Martínez C, Garcia-Vicuña R, Juarranz Y, Lamana A. et al., Vasoactive intestinal peptide in early spondyloarthritis: low serum levels as a potential biomarker for disease severity. J Mol Neurosci. 2015;56:577-84.

35. Jiang W, Gao SG, Chen XG, Xu XC, Xu M, Luo W, et al. Expression of synovial fluid and articular cartilage VIP in human osteoarthritic knee: a new indicator of disease severity? Clin Biochem. 2012;45:1607-12.

36. Leceta J, Gomariz RP, Martinez C, Carrión M, Arranz A, Juarranz Y. Vasoactive intestinal peptide regulates Th17 function in autoimmune inflammation. Neuroimmunomodulation. 2007;14:134-8.

37. Zúñiga LA, Jain R, Haines C, Cua DJ. Th17 cell development: from the cradle to the grave. Immunol Rev. 2013;252:78-88.
38. Yi A, Jian L, Xiaojing H, Hui X. The prevalence of Th17 cells in patients with dilated cardiomyopathy. Clin Invest Med. 2009;32:E144-50.

39. Li D, Morales A, Gonzalez-Quintana J, Norton N, Siegfried JD, Hofmeyer M, et al. Identification of novel mutations in RBM20 in patients with dilated cardiomyopathy. Clin Transl Sci. 2010;3:90-7.

40. Almeida MS, Lorena VM, Medeiros CA, Junior WO, Cavalcanti MD, Martins SM, et al. Alternative Th17 and CD4(+) CD25(+) FoxP3(+) cell frequencies increase and correlate with worse cardiac function in Chagas cardiomyopathy. Scand J Immunol. 2018;87:e12650.

41. Guedes PM, Gutierrez FR, Silva GK, Dellalibera-Joviliano R, Rodrigues GJ, Bendhack LM, et al. Deficient regulatory T cell activity and low frequency of IL-17-producing T cells correlate with the extent of cardiomyopathy in human Chagas' disease. PLoS Negl Trop Dis. 2012;6:e1630.

42. Reis PG, Ayo CM, de Mattos LC, Brandão de Mattos CC, Sakita KM, de Moraes AG, et al. Genetic polymorphisms of IL17 and Chagas disease in the South and Southeast of Brazil. J Immunol Res. 2017;2017:1017621. 